\title{
Exploring Constraint: Simulating Self-Organization and Autogenesis in the Autogenic Automaton
}

\author{
Stefan Leijnen*,**, Tom Heskes ${ }^{\dagger}$, Terrence W. Deacon ${ }^{\ddagger}$
}

\begin{abstract}
Origin of life theories often argue that molecular self-organization explains the spontaneous emergence of structural and dynamical constraints. However, the preservation of these constraints over time is not well-explained because of the self-undermining and self-limiting nature of these same processes. A process called autogenesis has been proposed in which negative structural coupling between self-organized processes preserves the constraints thereby accumulated. This paper presents a computer simulation of this process (the Autogenic Automaton) and compares its behavior to the same self-organizing processes when uncoupled. We demonstrate that this coupling produces a second-order constraint that can both resist dissipation and become replicated in new substrates over time.
\end{abstract}

Keywords Self-organization, origin of life, autogenesis, constraint, simulation, artificial chemistry

\footnotetext{
${ }^{*}$ Corresponding author

**Institute for Computing and Information Sciences, Radboud University Nijmegen, Heijendaalseweg 135, 6525 AJ Nijmegen, Netherlands. E-mail: stefan@leijnen.org, phone: +31.624375294.

${ }^{\dagger}$ Institute for Computing and Information Sciences, Radboud University Nijmegen, Heijendaalseweg 135, 6525 AJ Nijmegen, Netherlands. E-mail: t.heskes@ science.ru.nl, phone: +31.243652696.

${ }_{\ddagger}^{\ddagger}$ Department of Anthropology, University of California, 232 Kroeber Hall, Berkeley, CA 94720, USA. E-mail: deacon@ berkeley.edu, phone: +1.5106423392.
} 


\section{Introduction}

How could life have emerged? Although understanding life's origins does not necessarily imply understanding life as we find it around us today [6], it promises far reaching consequences for scientific fields such as cognitive science [31] and artificial intelligence [23] as the emergent dynamics that created life may have also shaped mind, machine and society.

Theories explaining the possible origins of life are as numerous as the properties that are claimed to define it. Some define life based on a general capacity for replication [34] or the exchange of energy and matter with an external environment [24, 29]. Others characterize life as systems using template-based encoding (e.g. RNA) [19] or being composed of autocatalytic sets [26]. Although each of these theories has its own flavor in explaining life's possible origins [21], many assume the involvement of the structure forming dynamics of self-organization.

Despite their often pivotal role in explaining the emergence of life, self-organizing processes are limited in their capacity to maintain structure [28]. A recently proposed theory suggests that, beyond mere self-organization, a synergetic coupling between self-organizing processes is a minimal requirement for life [11]. Through this higher-order linkage, the processes that generate structure may persistently recreate a capacity for self-creation, leading to robustness and a potential capacity for long-term sustenance and natural selection. As an example of such an autogenic process, a proto-life model system called the autogen is described to illustrate how two self-organizing processes - reciprocal catalysis and self-assembly - maintain each other's boundary conditions and thereby mutually increase the probability of persistence over time [10].

Currently, the autogen model is a theoretical proposition that remains to be validated experimentally. As a step toward validation, this paper describes a series of simulation experiments that investigate the self-organizing and autogenic properties of the autogen model. A simplified particle system simulation called the Autogenic Automaton models the synergetic linkage 
of self-organizing processes that leads to the emergence of autogens. In the next section this higher-order linkage is described in more detail, followed by a description of the computational model and the methods used to simulate and quantify the self-organizing and autogenic processes that generate, eliminate, and preserve constraint.

\section{Autogenesis}

Life's ability to resist degradation and persist in hostile environments is both ubiquitous and astonishing. Generation of structure, preservation by repair, and trait persistence through reproduction are perpetually organized in a continuous struggle against the destabilizing mechanisms of the second law of thermodynamics. In this section, autogenesis is introduced in three parts: first, how the inevitable increase of thermodynamic entropy poses a problem for models of life based on self-organization. Then autogenesis is presented as a three-tiered process hierarchy of constraint elimination, constraint generation and constraint preservation (and ensuing constraint selection), after which this hierarchy is exemplified by the autogen model, to be used for the subsequent simulation experiments.

\subsection{Second-Order Self-Organization}

Self-organization may occur in open systems that are continually perturbed toward a far-fromequilibrium state through incessant nonlinear amplification of local fluctuations [28]. These systems tend to reduce their statistical entropy, i.e. their variety of potential states, thereby becoming statistically less complex; for this reason, self-organization may also be regarded as self-simplification [1].

A typical example is the formation of Rayleigh-Bénard convection cells, which may emerge if a fluid is heated from below, causing fast-moving molecules in the bottom to rise upward while slow molecules simultaneously move downwards. These two vertical motions lead to 
horizontal heat exchange between upward and downward moving molecules, obstructing the dissipation of heat from its source to the surface. Under certain conditions (e.g. temperature, viscosity, shape and size of the surface) hexagonal convection cells develop that minimize the horizontal heat exchange and thereby maximize vertical heat dissipation [15]. As the number of potential system states is reduced by the emergence of these cells, the system becomes more ordered. Other examples include laser beams [16], where optical amplification results in spatial coherence; vortices, such as whirlpools and tornadoes; and autocatalytic chemical reactions, where the product of a reaction is also a catalyst for it, leading to a nonlinear reaction increase under particular proximity conditions [32].

The nonlinear amplification that is typical for self-organization tends to push the thermodynamic conditions for further propagation toward the unfavorable. This may occur up to a point where the system is no longer far-from-equilibrium and the local thermodynamic entropy increase comes to a halt. For example, in a reciprocally catalytic set reaction rates may increase exponentially as more and more catalysts are produced, up until the point when not enough reactants are available for further propagation, and the self-organizing process ends. Given the universal presence of self-organization in living systems, how can it be possible that order persists long enough for complex organisms to come about? It has been suggested that the answer may lie in the hierarchical organization of self-organizing processes [11].

When the product of an autocatalytic reaction enables a second autocatalytic reaction, which produces a reactant that enables the first (or a third, etc., as long as the causal chain is eventually closed), a so-called hypercycle emerges [13]. Hypercycles represent one possible way in which self-organizing processes, autocatalytic cycles in this case, may be linked together in a dynamical process hierarchy. However, with respect to preventing self-undermining this particular type of second-order self-organization does not provide a sufficient solution: each autocatalytic cycle that the hypercycle consists of represents a potential weakest link, which may cause the 
fragile hypercycle to break down entirely when reactants or energy for this particular cycle are no longer available.

Autogenesis requires another type of second-order self-organization where two (or more) self-organizing processes not only promote each other, but also where they act as a supportive environment if one of them breaks down, such that their self-undermining tendencies are reciprocally counteracted.

\subsection{Constraint}

The formation of crystals through self-assembly is a self-organizing process. The probability of particle detachment decreases with the number of adjacent crystal cells that keep a particle in place, causing a spatial difference between particle positions, such that at some locations there are many particles present while at other locations there are few particles to none. This difference, maintained by the attachment and detachment rates of the assembly process, may be viewed as a constraint on the spatial distribution of particles. More generally, a reduction of the variety of macroscopic states can be understood as a constraint producing process [9].

From a constraint-centric perspective, the global increase of entropy predicted by the second law of thermodynamics appears to run counter to the production of constraint caused by self-organization. Thermodynamic entropy increase spontaneously introduces noise into the system, as the probability of a random microscopic event inducing more order is lower than the probability of an event inducing less order (there are relatively few ordered states), thereby increasing the variety of macroscopic states. An example of such a constraint eliminating, noise inducing process is an ice cube melting in a soda drink: considering the possible states of water molecules in the glass, the number of states where only some of the molecules are arranged in a solid ice-cube is by far outnumbered by the number of states where they are all mixed up.

Living systems tend to produce constraint as well as preserve it against elimination. This 
capacity allows organisms, and lineages of organisms, to persist over long stretches of time. Following the type of second-order self-organization described above as autogenesis, constraint preservation is enabled by a juxtaposition of constraint producing processes, such that they actively support each other's persistence [8]. Whereas self-promoting self-organizing processes (e.g. hypercycles) tend toward self-undermining and ultimately a breakdown of the causal cycle, this reciprocally counteracting juxtaposition actively prevents self-undermining from taking place.

The relatively stability of these structural synergies allows for a simple type of natural selection to occur, as different kinds of synergies may co-exist within the same system. Some will be better suited to prevailing conditions than others and therefore have a better chance of sustaining themselves. This eventually leads to an elimination of noise (or reduction of variety) on a higher level, as unsuccessful noise-reducing synergies are removed. In this selection logic a discontinuity becomes apparent again, as the structured parts are separated in a competition caused by the dissipative potential of the whole. This transition represents a shift in logic comparable but opposite to the emergence of ordered structure from chaos: it could be argued that this shift constitutes a secondary kind of emergent transition between dynamical regimes.

\subsection{Autogen}

The three-tiered constraint hierarchy of autogenesis is exemplified by a minimal model system of life, the so-called autogen. In earlier publications (e.g. [10]) this model system was referred to as 'autocell'. However, since it exemplifies a general class of self-generating systems (rather than being typically cellular) 'autogen' is the preferred term. Autogens are formed by a synergistic relation between self-assembly and reciprocal catalysis, both being self-organizing processes. 



Figure 1: Illustration of autogen formation (left) and the reactions involved (right). Autogens constitute a dynamical linkage between self-assembly and reciprocal catalysis. In this model system, self-assembly is a self-organizing process where $G$ particles attach to one another, forming $G^{n}$ crystals of size $n$. These crystals may break up due to detachment. $G$ particles are generated by a reciprocally catalytic set of six different particle types ( $A$ to $F$ ). In turn, crystals may contain $C$ and $F$ particles, isolating these catalysts from potential reactants.

In a reciprocally catalytic system, each reaction initially leads to an increased probability for another reaction to take place as more and more catalysts are created. Exponential growth ensues until the reactants are depleted. Reciprocal catalysis leads to exponential increase of reactions that is limited solely by the number of available reactants. A boundary or container would prevent the exhaustion of reactants by removing them from the environment, thereby preserving a chemical potential for further dissipation [24]. Such a container may itself be formed by a self-organizing process, e.g. crystal growth through self-assembly [14]. Autogenesis, then, suggests that the form and function of a self-assembled container is dynamically linked to the autocatalytic process as it prevents the reactants from being depleted. Similarly, it explains how 
the autocatalytic process dynamically shapes the form and function of the crystals as it affects the process of self-assembly.

The autogen model is illustrated in figure 1. Particles of type $A$ and $B$ react to form a $C$ particle, catalyzed by particles of type $F$. A similar reaction takes place for particles of type $D$ and $E$, which form $F$ and $G$ particles, catalyzed by $C$ particles. In the same system, $G$ particles attach to one another creating self-assembled crystals of type $G^{n}$, with $n$ for the number of $G$ particles the crystal consists of. Due to their particular form, these crystals may contain catalysts, thereby negatively affecting the immediate production of $G$ particles but ensuring a potential for $G$-particle production over time. The negative structural coupling ensures that selfassembly stops before the catalysts are depleted, even though they are contained and therefore not readily available in the environment. This reaction potential is employed when a crystal opens up after detachment: contained catalysts are released, initiating a new chain of catalytic reactions that provides new $G$ particles used to repair the container, after which it may close again, thereby completing the work cycle.

The autogenic process as a whole then gains a minimal form of autonomy: as it is able to do work on its own conditions for sustenance, it grows independent from the conditions of its environment and becomes more dependent on its own internalized constraint. Under some conditions that are strived to be maintained, probability of growth and sustainment is higher than that of breakdown. When the autogen is damaged, it likely begins to repair itself. Autogenesis is about the higher-order constraint on the constraint generating processes of which it is constituted, such that a self is reproduced. It is a dynamic for the maintenance of itself as it maintains an implicit description of its units of preservation. 


\section{The Autogenic Automaton}

The logical steps building up to autogenesis, exemplified by the autogen model described above, are simulated with the Autogenic Automaton. Other computational proto-life models have simulated the formation of containers, the emergence of collectively autocatalystic sets, or both $[25,33]$. Although this simulation falls into the latter category, the goal here is not to provide a physically accurate model of either self-organizing process, nor of their synergy, but rather on demonstrating the viability of the logical hierarchy leading up this synergy.
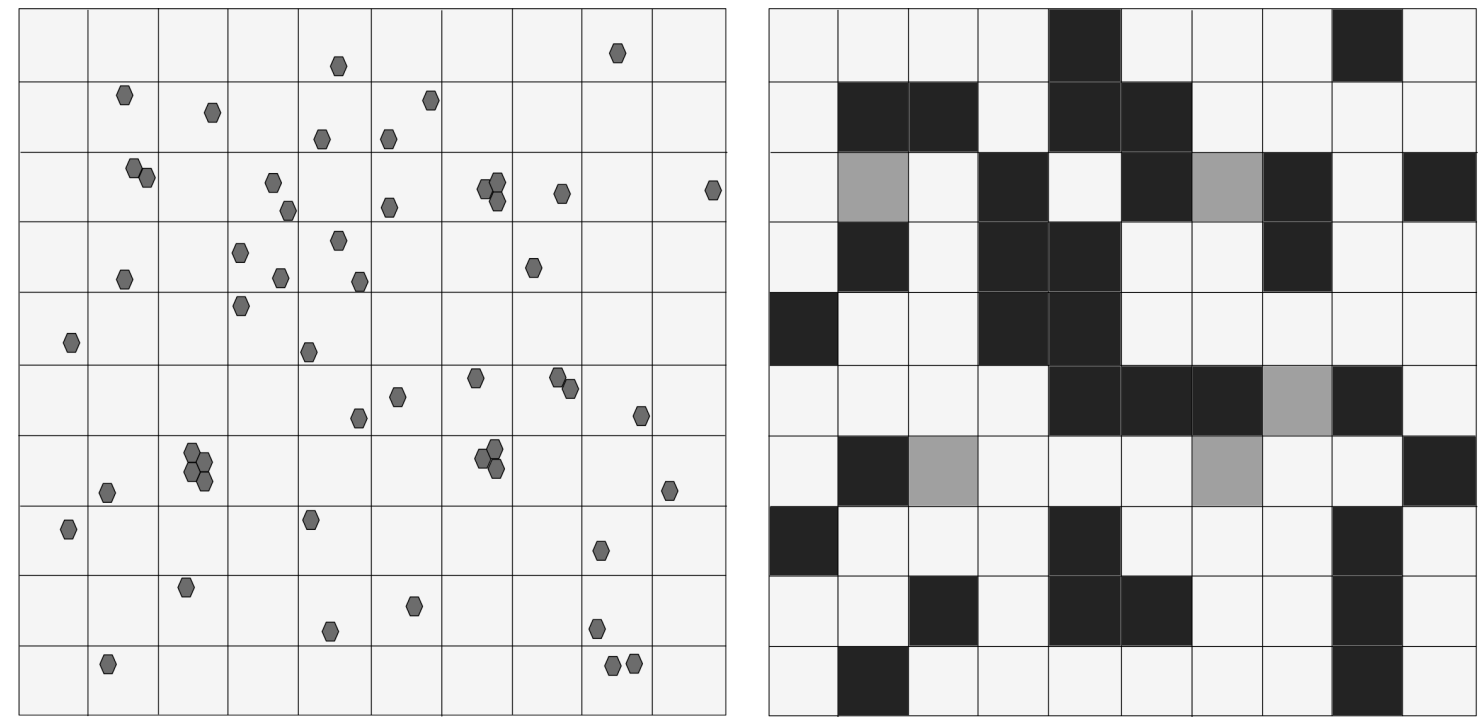

Figure 2: A continuous, closed particle system (left) is modeled in the Autogenic Automaton as a discrete grid of $10 \times 10$ tiles (right).

\subsection{Model}

A two-dimensional $10 \times 10$ tile grid is used as a discrete model of a closed reaction-diffusion system (fig. 2). Particle movement and the reaction rules that govern particle attachment, detachment, and the creation and removal of particles are all computated locally per tile. In this sense, the system resembles a cellular automaton allowing for emergence [4], where tiles may 
be occupied by multiple particles. Diffusion caused by particle-to-particle collisions is approximated by randomly directed movement to a horizontally or vertically neighboring tile with probability $P_{\text {movement }}=0.1$ at every time step, for each particle.

Particle-to-particle reactions and associated probabilities are modeled after crystallization and reciprocal catalysis. To this end, six particle types are defined and used for modeling reciprocal catalysis while a seventh type models the formation of crystals through self-assembly.

\subsection{Simulation}

The Autogenic Automaton is initialized by assigning random grid locations to a predefined quantity of particles of each type. Next, for a given number of time steps, new particle positions are computed and the reaction rules are applied to the particles at each tile.

One advantage of these localized reactions is that only small subsets of the total number of particles interact at each time step, reducing the computational complexity of the simulation. Another way to keep the model relatively simple and the simulation computationally tractable is to model only the aspects of self-organization that are necessary for showing the viability of autogenesis, which include nonlinear probability functions and reversible reactions. Other physical properties typical for particle systems (e.g. kinetic energy, dissipation of heat) are not modeled.

In order to show the generation and elimination of constraint (i.e. macroscopic change) over time, the simulation starts in non-equilibrium conditions. Due to the absence of heat and friction, the entropy potential necessary for far-from-equilibrium systems to be self-organizing is not defined with respect to thermodynamic equilibrium (when all movement and reactions have ceased to occur) but rather with respect to chemical equilibrium. Thus, the initial set of

particles is not in chemical equilibrium since no crystallization has occured nor has a catalytic reaction taken place. So, the macrosopic change that is observed in the experiments that follow 
may be attributed to the system moving toward a chemical equilibrium.

\subsection{Quantifying Constraint}

Through the course of a simulation run, particles move and collide against one another to create new particles or crystals, or they fall apart. The system moves through various macroscopic states, caused or maintained by processes that generate, eliminate, preserve or select constraint; the quantification of statistical entropy described here yields an indirect observation of the underlying processes.

Constraint is expressed by means of the information entropy over the spatial probability distribution of particular event types in the tile grid [20, 17, 27]. Notable characteristics of the particle system surface by observing the (in-)homogeneity of the locations of events that correspond to these characteristics, such as the presence of a particle type or the occurrence of a specific reaction.

Given a set of probabilities $p_{i}$ for $i=1, \ldots, n_{\text {tiles }}$ with $0 \leq p_{i} \leq 1$ and $\sum_{i} p_{i}=1$, information entropy is defined as $[30,7]$ :

$$
H=-\sum_{i=1}^{n_{\text {tiles }}} p_{i} \log _{2} p_{i} .
$$

Now, when we consider an event $X$, we substitute

$$
p_{i}=\frac{\left|X_{i}\right|}{|X|}
$$

with $\left|X_{i}\right|$ the number of events at tile $i$ and $|X|=\sum_{i}\left|X_{i}\right|$ the total number of events, to obtain

$$
H(X)=-\sum_{i=1}^{n_{\text {tiles }}} \frac{\left|X_{i}\right|}{|X|} \log _{2} \frac{\left|X_{i}\right|}{|X|} .
$$

For ease of interpretation we often consider the so-called normalized information entropy [18] 


$$
\hat{H}(X)=-\frac{1}{\log _{2} n_{\text {tiles }}} \sum_{i=1}^{n_{\text {tiles }}} \frac{\left|X_{i}\right|}{|X|} \log _{2} \frac{\left|X_{i}\right|}{|X|},
$$

which normalizes the standard information entropy by its maximum value such that always $0 \leq \hat{H}(X) \leq 1$. For a completely homogeneous distribution of events over tiles we now have $\hat{H}(X)=1$, whereas $\hat{H}(X)=0$ when all events $X$ are concentrated at a single tile.

\subsection{Quantifying Multiple Constraint Types}

In some cases it is necessary to measure the spatial difference between two event types, $X$ and $Y$, using the Kullback-Leibler divergence of their distributions over the grid tiles [22]:

$$
D_{K L}(P \| Q)=\sum_{i=1}^{n_{\text {tiles }}} p_{i} \log _{2} \frac{p_{i}}{q_{i}} .
$$

Substituting $p_{i}$ and $q_{i}$ with $\frac{\left|X_{i}\right|}{|X|}$ and $\frac{\left|Y_{i}\right|}{|Y|}$, respectively, would yield infinite divergence for a distribution with a tile $i$ such that $\left|X_{i}\right|>0$ and $\left|Y_{i}\right|=0$. To resolve this problem, a smoothing function is used [3], where

$$
q_{i}=\left\{\begin{array}{r}
\alpha \frac{\left|Y_{i}\right|}{|Y|} \text { for }\left|Y_{i}\right|>0 \\
\epsilon \text { for }\left|Y_{i}\right|=0
\end{array},\right.
$$

with $\epsilon=10^{-5}$ and normalization coefficient $\alpha$ chosen such that the probabilities sum to 1 . $p_{i}$ is substituted similarly with $\frac{\left|X_{i}\right|}{|X|}$. For ease of exposition, we will omit this smoothing in subsequent formulas.

\section{Constraint Generation}

The Autogenic Automaton is used to simulate the generation of constraint in two separate selforganizing processes: the formation of crystals through self-assembly, and the local amplification of reactions taking place in a reciprocally catalytic set. 


\subsection{Self-Assembly}

Self-assembly is modeled by a series of attachment and detachment reactions between $G$ particles and crystals $G^{n}$ with reaction parameters $\gamma^{+}$and $\gamma^{-}$:

$$
G^{n}+G \rightleftarrows G^{n+1}
$$

with $n \geq 1$ and $G^{1} \equiv G$. If a $G$ particle is located within the same tile as either another $G$ particle or crystal, the probability of attachment is given by

$$
P_{g}^{+}=\gamma^{+} \in[0,1]
$$

Modelling detachment as the opposite of attachment, crystals have a probability $P_{g}^{-}$of a $G$ particle detaching from the crystal. Larger crystals are more tightly connected and less likely to break apart than smaller crystals due to a larger number of kinks holding the individual particles together [5]. An increased size yields a lower probability of detachment and therefore increases the probability for further growth. This introduces a nonlinearity in the crystal growth process, reflected in our model system by a detachment probability function that is negatively exponential to the crystal size $n$.

$$
P_{g}^{-}=\left(1+\exp \left[\gamma^{-}\right]\right)^{-n}
$$

with $\gamma^{-} \in \mathbb{R}, n \geq 2$. Following equation (3.1), event $X_{i}$ is defined with respect to self-assembly as the observation of a $G$ particle at tile $i$, where $G^{n}$ crystals are counted as $n$ observations. Therefore, the generation of constraint during this process is examined using

$$
\hat{H}(G, t)=-\frac{1}{\log _{2} n_{\text {tiles }}} \sum_{i=1}^{n_{\text {tiles }}} \frac{\left|G_{i}(t)\right|}{|G(t)|} \log _{2} \frac{\left|G_{i}(t)\right|}{|G(t)|},
$$

for the normalized information entropy of $G$ at time $t$. 




Figure 3: Decrease of normalized information entropy $\hat{H}(G, t)$ during self-assembly of 1000 $G$ particles with $\gamma^{+}=1$, for several detachment probabilities: $-\gamma^{-}=-5$, $\gamma^{-}=-3, \cdots \gamma^{-}=-4$, $\gamma^{-}=-1$. The images on the right depict how $\hat{H}(G, t)$ correlates with the distribution of $G$ particles over the grid, ranging from an almost homogeneous distribution (top) to a few $G^{n}$ crystals (bottom). Results are averaged over 100 trial runs.

Figure 3 shows the development of $\hat{H}(G, t)$ over time, for different values of $\gamma^{-}$. With $\gamma^{-}=-5$ the probability of detachment $P_{g}^{-}$is relatively high, such that many crystals fall apart and the constraint on particle $G$ locations is low. With $\gamma^{-} \in[-1,-2], P_{g}^{-}$is relatively low: once formed, crystals do not break apart, leaving no single $G$ particles for attachment and no room for further growth. The $G$ particle locations are maximally constrained for $\gamma^{-}=-4$. 


\subsection{Reciprocal catalysis}

Particle types $A$ to $F$ are used to model self-organization through reciprocal catalysis. Particles of type $A$ and type $B$ may react to form a $C$ particle when both are located in the same tile; similarly for particles $D$ and $E$ forming an $F$ :

$$
\begin{aligned}
& A+B \stackrel{F}{\rightleftarrows} C, \\
& D+E \stackrel{C}{\rightleftarrows} F .
\end{aligned}
$$

Particles $F$ and $C$ are catalysts for reactions (4.2) and (4.3), respectively. Reaction probability $P_{r}^{+}$increases exponentially with $n$, the number of catalysts present at the same tile $i$ (i.e. $n=$ $\left|F_{i}\right|$ for the former reaction, and $n=\left|C_{i}\right|$ for the latter)

$$
P_{r}^{+}=\left(1+\exp \left[\varrho^{+}\right]\right)^{-(1+n)^{-2}},
$$

with $\varrho^{+} \in \mathbb{R}$. The reverse reactions $(C$ particles splitting into $A$ and $B$ particles and $F$ into $D$ and $E$ ) occurs with probability $P_{r}^{-}$for every $C$ or $F$ particle, at each time step

$$
P_{r}^{-}=\varrho^{-} \in[0,1] .
$$

Similar to self-assembly, reciprocal catalysis is a locally nonlinear process: one catalytic reaction increases the likelihood of another catalytic reaction occuring. However, the observable artifacts of reciprocal catalysis (i.e. the produced catalysts) may cease to exist once this amplification process no longer takes place, or they may diffuse to different locations. To quantify the amount of constraint generated, we therefore use the probability distribution of reaction locations as observed events, rather than the catalysts themselves. 


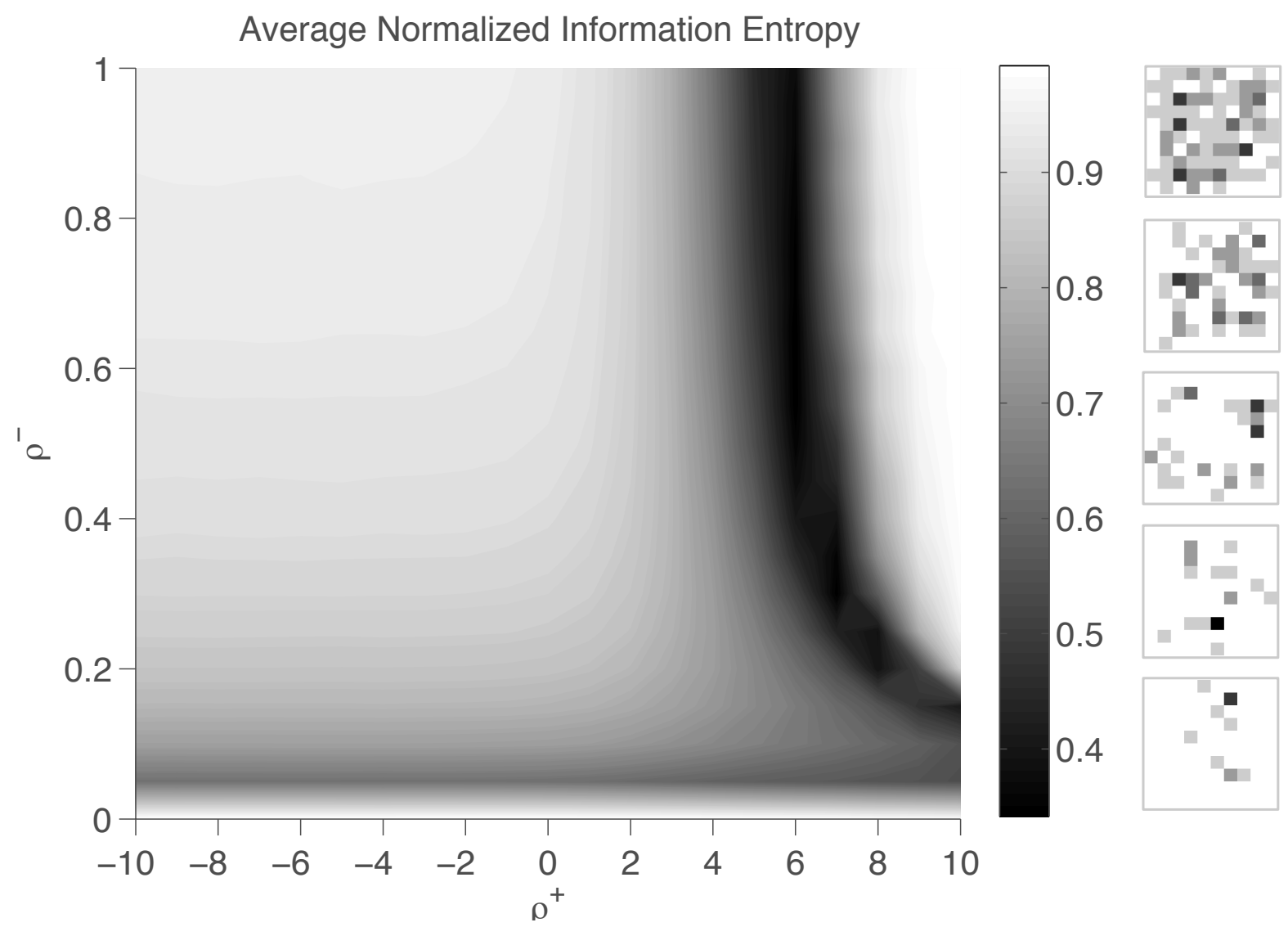

Figure 4: Normalized information entropy during reciprocal catalysis, after initialization with 1000 particles distributed equally among types $A, B, D$, and $E$. For given $\varrho^{+}$and $\varrho^{-}, \hat{H}(R, t)$ is averaged over 5000 time steps and 10 trial runs. The right-side images depict the distribution of catalytic reactions over the grid.

The constraint generated by reciprocal catalysis is quantified by a decrease in normalized information entropy over the distribution of catalytic reaction loci $R$ at time $t$ :

$$
\hat{H}(R, t)=-\frac{1}{\log _{2} n_{\text {tiles }}} \sum_{i=1}^{n_{\text {tiles }}} \frac{\left|R_{i}(t)\right|}{|R(t)|} \log _{2} \frac{\left|R_{i}(t)\right|}{|R(t)|} .
$$

In order to investigate the effect of parameters $\varrho^{+}$and $\varrho^{-}$on the normalized information entropy, $\hat{H}(R, t)$ is averaged over time: 


$$
\frac{1}{t_{\max }} \sum_{t=1}^{t_{\max }} \hat{H}(R, t) .
$$

Results for $t_{\max }=5000$ are shown in figure 4 . It is found that distribution $R$ is most constrained for $\varrho^{+} \approx 6$ and $\varrho^{-}>0.5$ (i.e. catalysts break up regularly) .

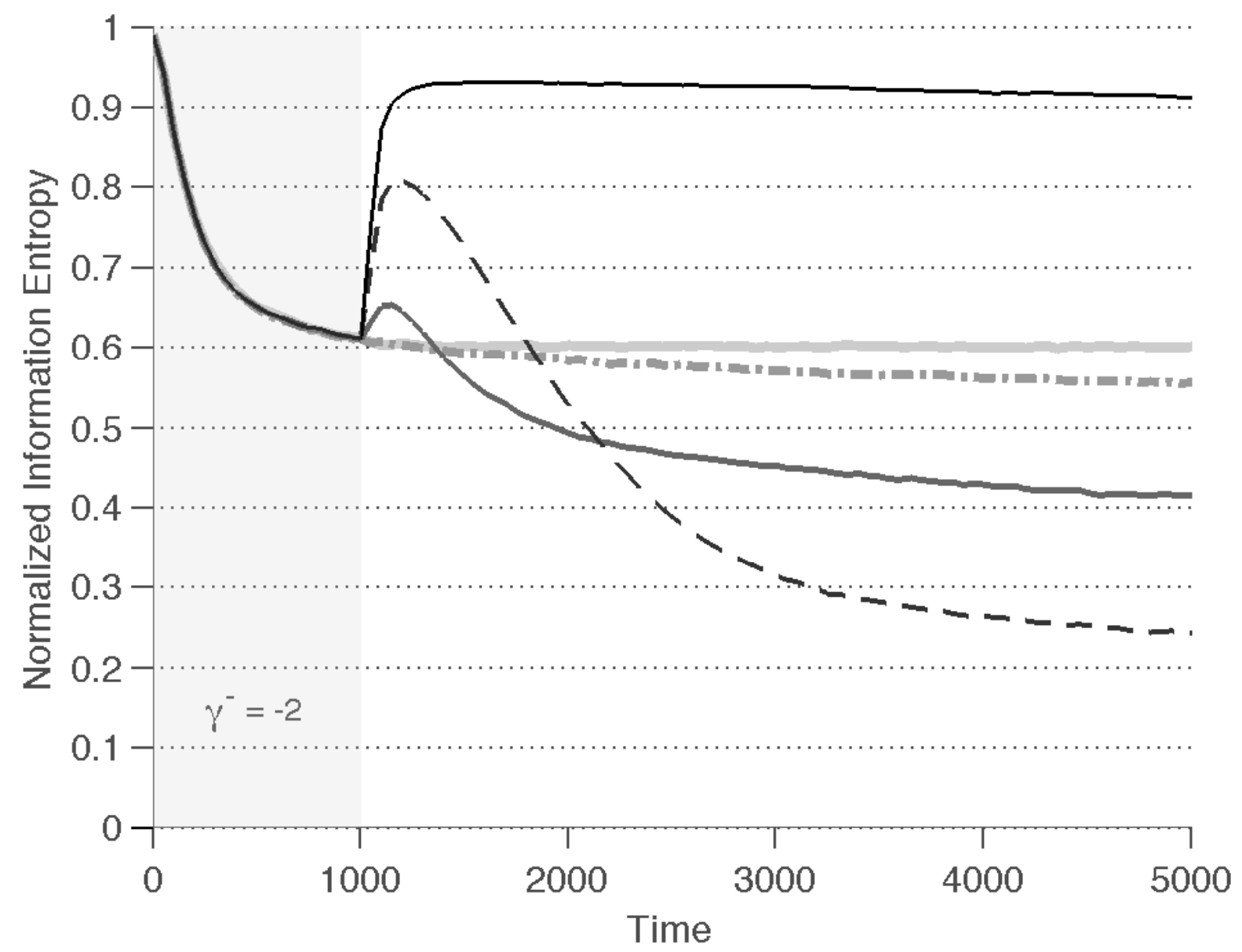

Figure 5: Elimination of constraint: the Autogenic Automaton is initialized with $1000 G$ particles and $\gamma^{+}=1, \gamma^{-}=-2$. At $t=1000, \gamma^{-}$is changed: $-\gamma^{-}=-5$, $\gamma^{-}=-3,-\gamma^{-}=-4, \square \gamma^{-}=-1$. For $\gamma^{-} \in[-3,-4]$ the eventual decrease in information entropy is preceded by an initial increase. Results are averaged over 100 trials. 


\section{Constraint Elimination}

Self-organizing processes are enabled by specific conditions that promote local amplifications. Due to change initiated by self-undermining (or imposed externally) the boundaries of these conditions may be transgressed leading to an elimination of previously generated constraint. This process can be demonstrated by means of a so-called process spectometry: after an initial phase with conditions that enable self-assembly, the value of $\gamma^{-}$is changed, and the effect on the normalized information entropy is observed (fig. 5).

The figure shows that after $t=1000$ the normalized information entropy is (initially) higher for $\gamma^{-} \in[-5,-3]$. After some time this value decreases as constraint grows: $G$ particles that have previously detached from small crystals now attach to others, such that only large crystals remain. Within the 5000 time steps shown, this effect results in higher local concentrations of $G$ particles, less homogeneity, a lower value of $\hat{H}(G, t)$ and more constraint for $\gamma^{-} \in[-4,-3]$.

\section{Constraint Preservation}

The higher-order linkage between self-assembly and reciprocal catalysis has been discussed in section 2.3, where a negative structural coupling is suggested that works in two directions:

1. $G$ particles generated by autocatalysis are created in close proximity to one another, due to the locality of the catalytic amplifications, thereby increasing the likelihood of crystal growth;

2. crystals may act as containers for catalysts, preventing the reciprocally catalytic process from undermining itself and preserving a potential for catalysis at a later point in time.

Modeling this synergetic linkage, reaction 4.3 is changed as follows:

$$
D+E \stackrel{C}{\rightarrow} F+G
$$


This increases the likelihood of $G$ particles being produced in close proximity to a catalytic reaction. For the reverse linkage a crystal needs to be capable of containing catalysts upon formation, to be released again when the crystal breaks up. Therefore reaction 4.1 is changed, where a crystal of size $n$ containing $k C$ particles and $m F$ particles is denoted as $G^{n}(k C, m F)$ :

$$
\begin{gathered}
G^{n}(k C, m F)+G+p C+q F \rightarrow G^{n+1}((k+p) C,(m+q) F), \\
G^{n}(k C, m F) \rightarrow G^{n-1}+G+k C+m F,
\end{gathered}
$$

with $k, m, p, q \geq 0$ and $n \geq 2$. The continuous addition of $G$ particles is balanced by removing $G$ particles at each time step with probability $P_{g}^{-}=\left(1+\exp \left[\gamma^{-}\right]\right)^{-1}$.

\subsection{Parametrization}

Thus far, four reaction parameters have been introduced: $\gamma^{+}, \gamma^{-}, \varrho^{+}$and $\varrho^{-}$. The results in section 4.1 showed that a decrease in $\hat{H}(G, t)$ may occur when $\gamma^{+}$is fixed at 1 . Similarly, figure 4 shows that $\varrho^{-}>0.5$ allows for relatively low values of $\hat{H}(R, t)$. Given these values, we investigate the ranges of $\gamma^{-}$and $\varrho^{+}$that allow for both types of self-organization to occur simultaneously.

The redundancy between the distributions of $G$ particle locations and catalytic reactions is considered to be an indication of the amount of interaction between both processes, i.e. it measures whether crystals tend to be located in proximity to catalytic reactions, and vice versa. This is quantified using the Kullback-Leibler divergence with smoothing (eq. 3.2), which is symmetrized to obtain a commutative measure:

$$
\begin{gathered}
S D_{K L}(G, R, t)=D_{K L}(G(t)|| R(t))+D_{K L}(R(t)|| G(t)) \\
=\sum_{i=1}^{n_{\text {tiles }}}\left[\frac{\left|G_{i}(t)\right|}{|G(t)|}-\frac{\left|R_{i}(t)\right|}{|R(t)|}\right] \log _{2}\left[\frac{\left|G_{i}(t)\right|}{|G(t)|} / \frac{\left|R_{i}(t)\right|}{|R(t)|}\right],
\end{gathered}
$$



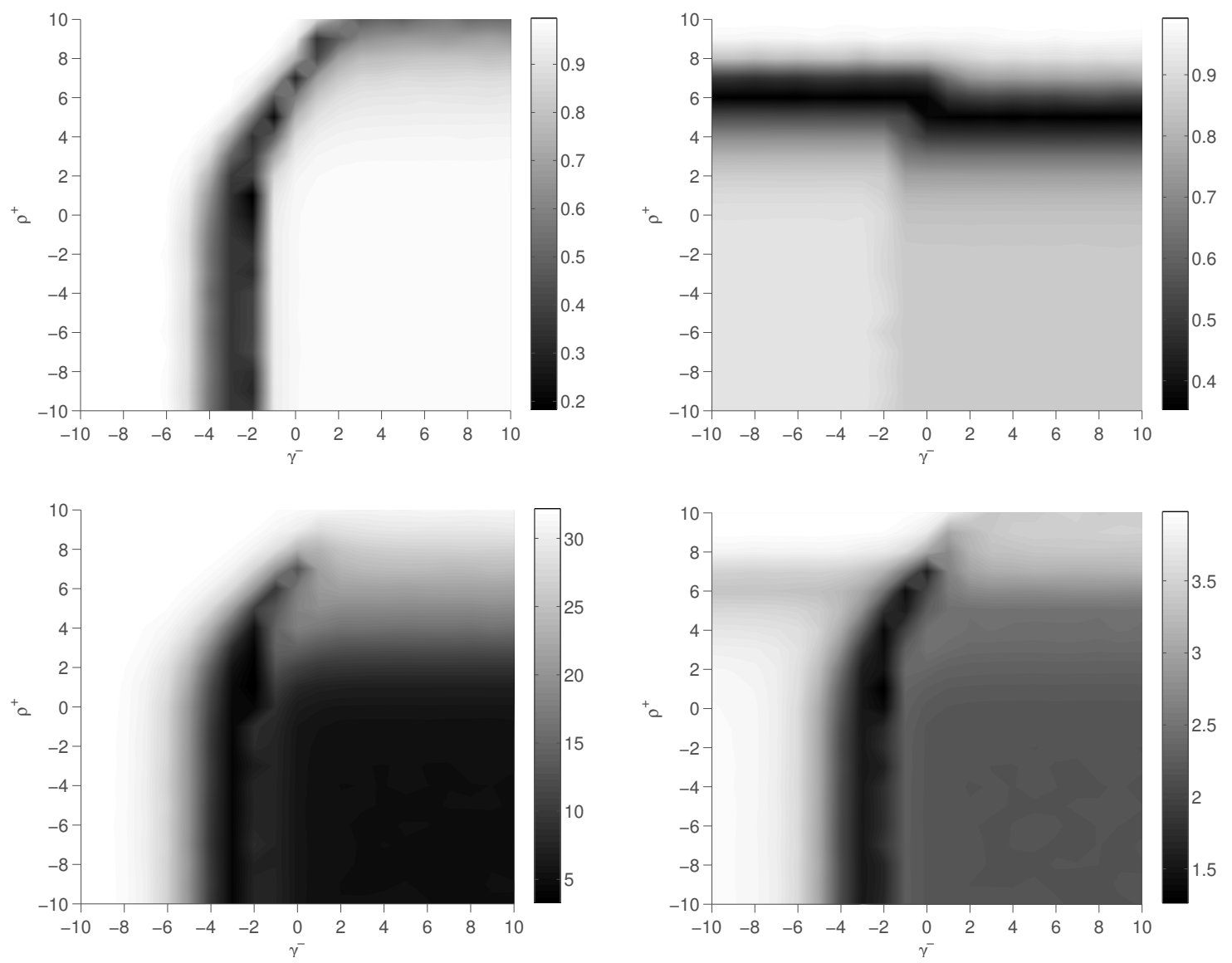

Figure 6: After initializing the simulation with 1000 particles evenly distributed among types $A$, $B, D$ and $E$, it is run for 5000 time steps with $\gamma^{+}=1, \varrho^{-}=0.5$ and $\gamma^{-}, \varrho^{+} \in[-10,10]$. The four figures above show the average normalized information entropy of $G$ particle locations (top left), the average normalized information entropy of catalytic reaction locations (top right), the average symmetrized Kullback-Leibler divergence with smoothing, where $S D_{K L}\left(\gamma^{-}, \varrho^{+}\right)=$ $\max \left(S D_{K L}\right)$ if no occurences are found (bottom left), and the sum of these three figures, where $S D_{K L}$ has been normalized using scaling coefficient $\beta$ (bottom right). 
with smoothing (eq. 3.2) applied if necessary. Running constraint preservation experiments requires parametrization of $\gamma^{-}$and $\varrho^{+}$such that

(a) self-assembly takes place (i.e. $\hat{H}(G, t)$ is low);

(b) reciprocal catalysis takes place (i.e. $\hat{H}(R, t)$ is low);

(c) both processes take place in each other's proximity (i.e. $S D_{K L}(G, R, t)$ is low).

Figure 6 shows these three measurements averaged over 5000 time steps for different values of $\gamma^{-}$and $\varrho^{+}$. The desired parameter values are estimated by minimizing

$$
\hat{H}(G, t)+\hat{H}(R, t)+\beta S D_{K L}(G, R, t),
$$

where coefficient $\beta$ scales $S D_{K L}(G, R, t)$ to $[0,2]$

$$
\beta=2\left(\max _{\gamma^{-}, \rho^{+} \in[-10,10]} S D_{K L}(G, R, t)\right)^{-1},
$$

such that the normalized informaties entropies and the divergence between the distributions contribute equally to the sum.

\subsection{Preservation}

Using the parameter values found, a process spectrometry is generated (fig. 7). For $\gamma^{-} \in$ $[-5,-4]$ after $t=1000$, the high probability of detachment is not conducive for the prolonged persistence of crystals, and they fall apart. For $\gamma^{-}$remaining at $0, \hat{H}(G, t)$ continues to develop unperturbed. Changing $\gamma^{-}$to -1 results in a lower normalized information entropy, as more $G$ particles detach and subsequently attach to larger crystals (cf. fig. 3). With $\gamma^{-}$changed to $-2, \hat{H}(G, t)$ initially drops, but eventually finds a new equilibrium at a higher value than with $\gamma^{-} \in[-1,0]$. 

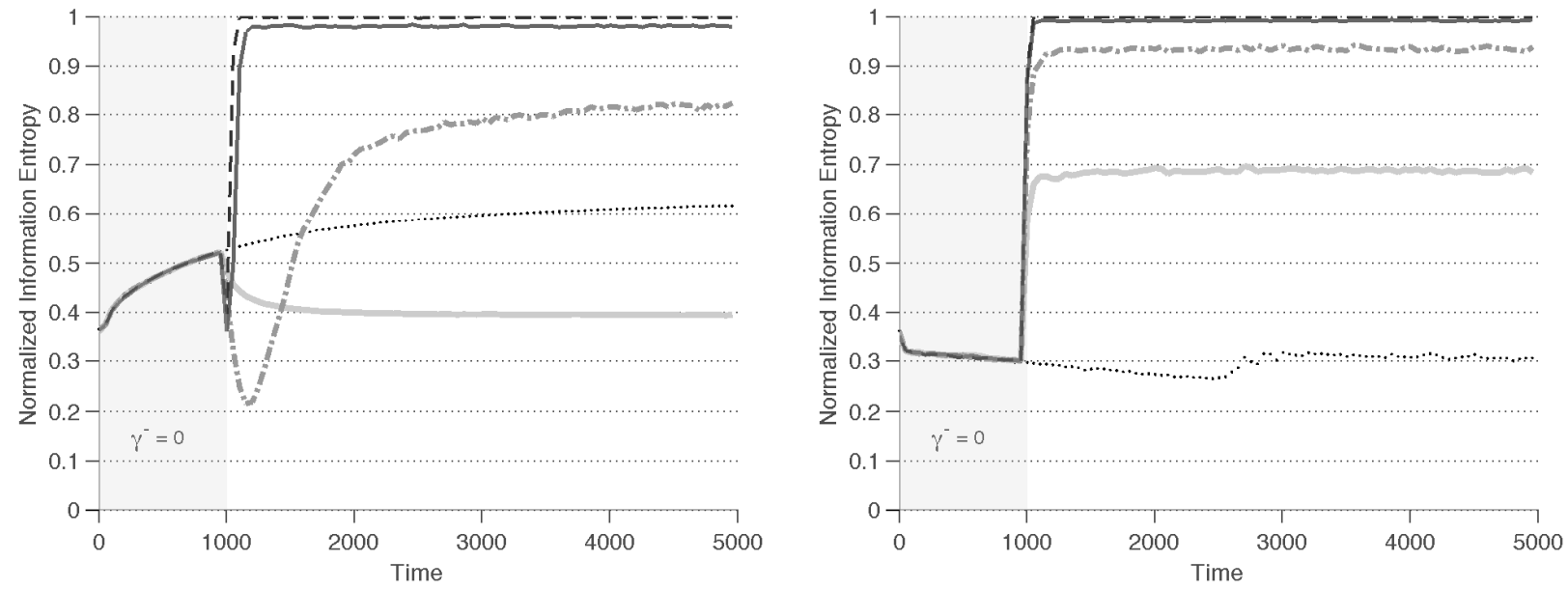

Figure 7: Normalized information entropy $\hat{H}(G, t)$ during autogenesis (left) and self-assembly without reciprocal catalysis where instead of being generated by a catalytic reaction, $G$ particles are added to random grid locations with the same rate as in the previous simulation (right). With $\gamma^{+}=1, \varrho^{+}=6, \varrho^{-}=0.5$, and $\gamma^{-}=0$ for $t \in[0,1000]$, and 1000 particles evenly distributed among types $A, B, D$ and $E$ initially. For $t \in[1001,5000],-{ }^{---} \gamma^{-}=-4$,

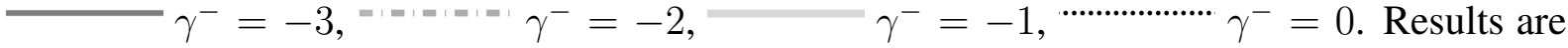
averaged over 1000 trials.

By itself, this figure provides limited insight into the processes that underly the production, elimination and preservation of constraint. However, it may be compared against a similar experiment that lacks a higher-order linkage. Since $\hat{H}(G, t)$ only measures the constraint of the distribution of $G$ particles, the experiment is repeated without the set of particles necessary for reciprocal catalysis. During the process spectometry of figure 7 (left), the number of $G$ particles that were added at each time step is stored. In this second experiment, $G$ particles are created at random grid locations at exactly the same rate. This allows for self-assembly to take place under similar circumstances, but decoupled from reciprocal catalysis. $G$ particle creation occurs at the same rate, although spatial proximity is no longer biased by reciprocal catalysis, and the absence of catalysts excludes the possibility of containment.

The results of this self-assembly experiment are shown in figure 7 (right). Comparing both 
figures, it is found for $\gamma^{-}=-2$ that constraint is preserved when a synergetic linkage between self-assembly and reciprocal catalysis is present while it largely falls apart in the case of mere self-assembly. Also for $\gamma^{-}=-1$, the value of $\hat{H}(G, t)$ remains lower with this linkage than without. This is not the case when $\gamma^{-}$remains at 0 . Here, self-assembly results in a more constrained system than when combined with reciprocal catalysis.

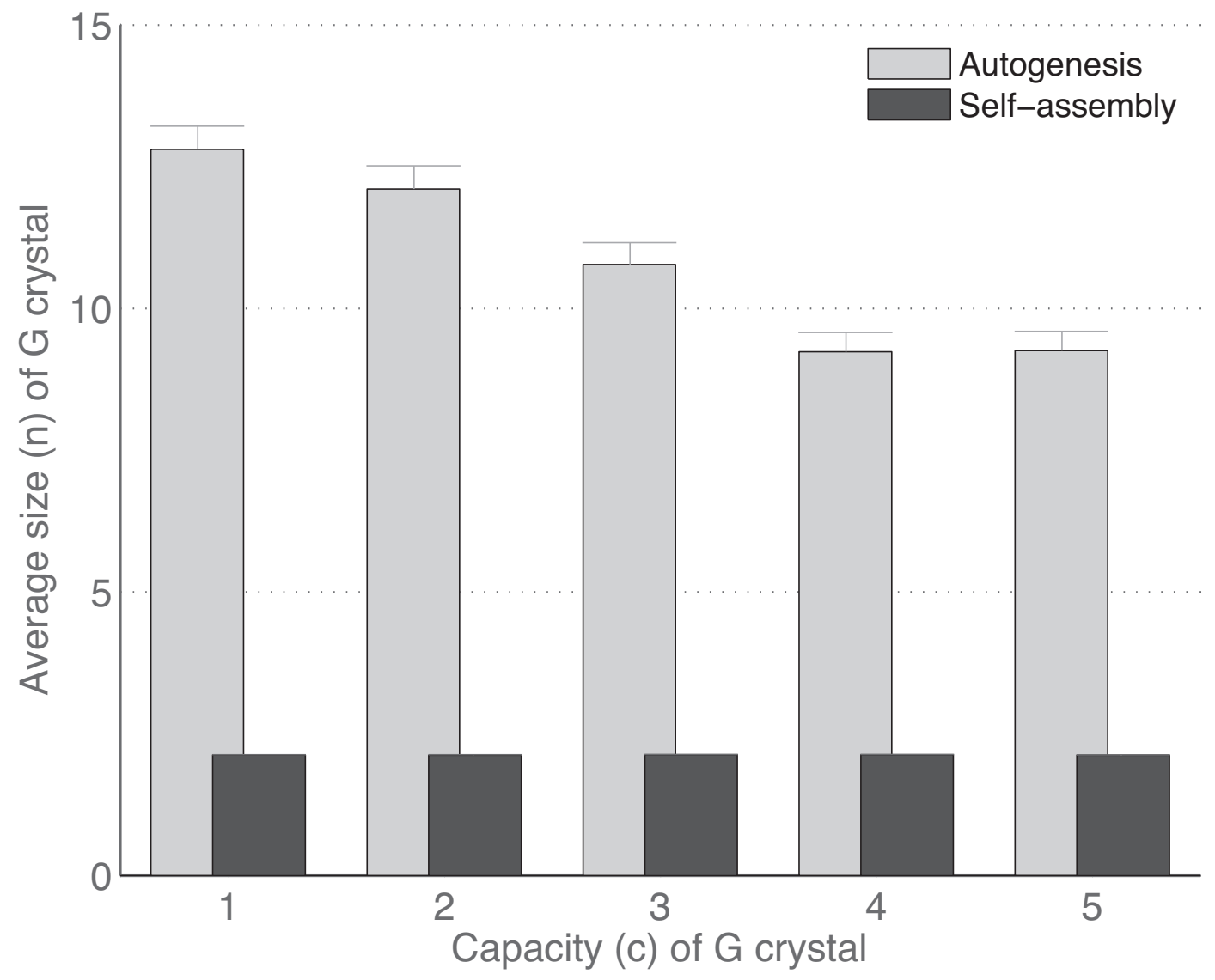

Figure 8: Fixing $\gamma^{-}$at -0.5 and $\gamma^{+}=1, \varrho^{+}=6$, and $\varrho^{-}=0.5$, the grid is again initialized with 1000 particles of types $A, B, D$ and $E$. For 1000 trial runs over 5000 time steps, the mean crystal size and standard error of the mean are reported for the five different containment capacities. 


\section{Constraint Selection}

The preservation of constraint is a higher-order process: not only is noise reduced as the spatial distribution of events becomes more constrained, but the distribution over specific constraint types, e.g. the specific form of crystals, is itself also reduced.
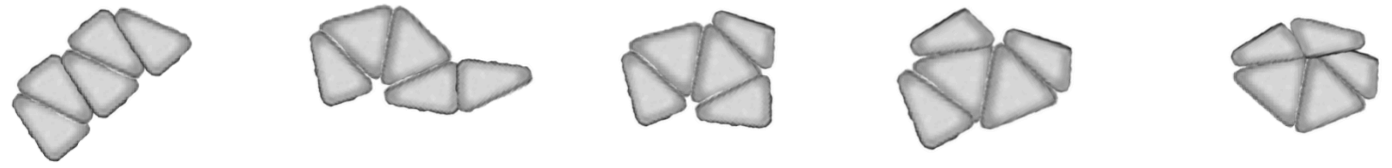

Figure 9: Due to the way $\mathrm{G}$ particles attach to one another, crystals with specific topologies may come about. This illustration shows several crystals $G_{c}^{n}$ of equal size (i.e. $n=5$ ) but with different capacities $(c)$ for containing catalysts, due their specific form.

In order to experimentally show the emergence of such higher-order constraint, the containment capacity of crystals is limited by their specific form (fig. 9). This is reflected in the Autogenic Automaton by initializing each new crystal with property $c$, a random value ranging from 1 to 5 that indicates the maximum number of catalysts a crystal may contain.

Figure 8 shows the average size of crystals compared against their maximum containment capacity, for both autogenesis and self-assembly without reciprocal catalysis. The results reveal two differences. First, a difference in crystal size between autogenesis and self-assembly, which could have been inferred from the results of the previous constraint preservation experiments.

The second difference is that, for autogenesis, a crystal's containment capacity is correlated with its average size. In our model system the removal probability $P_{g}^{-}$is independent of the specific crystal form, so the maximum containment of a crystal does not affect its size directly. Rather, the value of $c$ indirectly affects the growth of crystals, as the numbers of catalysts present at a tile will affect the production of new $G$ particles, and thereby a crystal's capacity for formation (or reconstitution) if those catalysts are released upon detachment. This work cycle 
creates a difference in size between the different crystal topologies, which is maintained despite the independence between crystal topology and the underlying self-organizing processes.

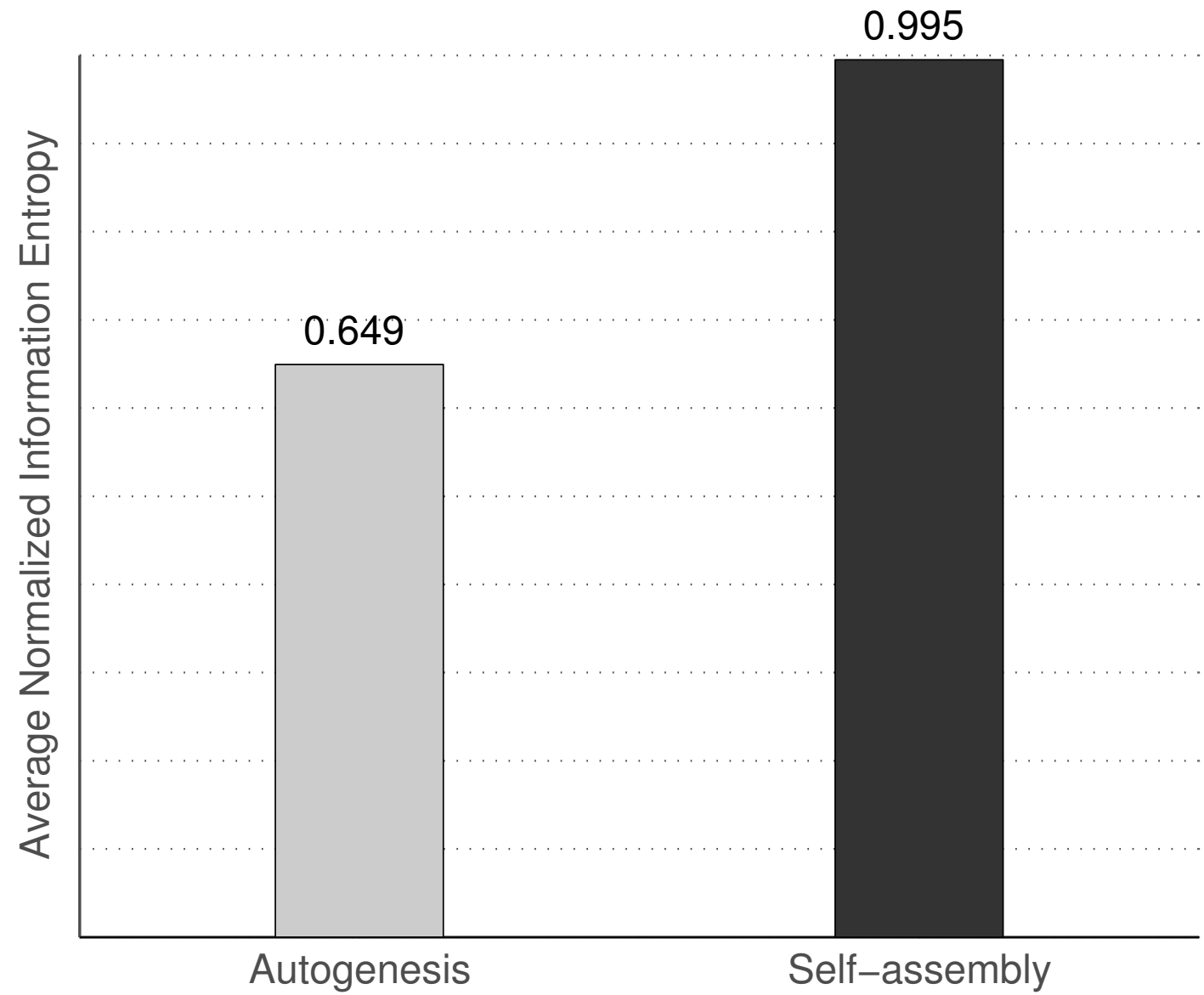

Figure 10: Higher-order constraint: the average normalized information entropy over the distribution of crystal capacities $\hat{H}\left(G_{c}^{n}, t\right)$ is substantially lower for autogenesis than for selfassembly without reciprocal catalysis. Results averaged over 1000 trial runs.

This higher-order constraint may be quantified using the normalized information entropy over the distribution of the containment capacities of crystals. With $p_{c}$ the probability that a crystal has a containment capacity $c,\left|G_{c}^{n}\right|$ the number of crystals with capacity $c$ and $\left|G^{n}\right|=\sum_{c}\left|G_{c}^{n}\right|$ the total number of crystals, 


$$
\begin{gathered}
p_{c}=\frac{\left|G_{c}^{n}\right|}{\left|G^{n}\right|}, \\
\hat{H}\left(G_{c}^{n}, t\right)=-\frac{1}{\log _{2} 5} \sum_{c=1}^{5} \frac{\left|G_{c}^{n}(t)\right|}{\left|G^{n}(t)\right|} \log _{2} \frac{\left|G_{c}^{n}(t)\right|}{\left|G^{n}(t)\right|} .
\end{gathered}
$$

Figure 10 shows the average $\hat{H}\left(G_{c}^{n}, t\right)$ over the simulation runs of the previous experiment for self-assembly and autogenesis. Selection of crystal topologies induced by specific conditions accounts for the relatively low normalized information entropy over containment capacities during autogenesis.

\section{Conclusions}

Constraint generation, elimination, preservation and selection have been shown to occur in the Autogenic Automaton simulation under particular conditions. Taken together, these processes constitute self-organization and autogenesis, albeit in a minimal sense. The self-undermining tendency of self-assembly and reciprocal catalysis is limited by virtue of their second-order synergy - a higher-order constraint on these constraint generating processes - leading to preservation of autogens and ultimately selection of crystal topology.

The experimental explorations described in this paper are not intended to quantify autogenesis, or to give a full account of autogenic properties and phenomena. Rather, they serve to demonstrate (1) the dynamics reversal that takes place in second-order self-organization, and (2) the higher-order noise reduction constituted by formal type selection that may emerge from competition between simple autogens. Those properties are what sets autogenic systems apart from other models of the emergence of proto-life. 
The hierarchical distinction between different types of constraint is reminiscent of a logicaltype distinction. Although the three statistical distributions used here ( $G$ particle locations, $R$ reaction locations, and $G_{c}^{n}$ containment capacities) are all subject to quantification in terms of information entropy, this quantification does not distinguish between physico-chemical constraints expressed by the former two, and substrate independent, formal constraint expressed by the latter. Since the physics underlying the maintenance of far-from-equilibrium states have largely been ignored in this simulation (cf. [2]), further research and simulation is required to develop tools capable of expressing this dynamical difference [12].

\section{References}

1. Ashby, W. R. (1957). An introduction to cybernetics. London: Chapman \& Hall Ltd.

2. Beer, R. D. (2004). Autopoiesis and cognition in the Game of Life. Artificial Life, 10(3), 309-326.

3. Bigi, B. (2003). Using Kullback-Leibler distance for text categorization. In Proceedings of the 25th European Conference on IR Research, ECIR'03. Berlin, Heidelberg: SpringerVerlag, (pp. 305-319).

4. Bilotta, E., \& Pantano, P. (2005). Emergent patterning phenomena in 2D cellular automata. Artificial life, 11(3), 339-362.

5. Burton, W. K., \& Cabrera, N. (1949). Crystal growth and surface structure. Part I. Discuss. Faraday Soc., 5, 33-39.

6. Cleland, C. E. (2013). Conceptual challenges for contemporary theories of the origin(s) of life. Current Organic Chemistry, 17, 1704-1709.

7. Cover, T. M., \& Thomas, J. A. (1991). Elements of Information Theory. Wiley. 
8. Deacon, T. W. (2003). The hierarchic logic of emergence: Untangling the interdependence of evolution and self-organization. In B. H. Weber, \& D. J. Depew, eds., Evolution and Learning: The Baldwin Effect Reconsidered. Cambridge, MA: MIT Press, (pp. 273-308).

9. Deacon, T. W. (2006). Emergence: The hole at the wheel's hub. In P. Clayton, \& P. Davies, eds., The Re-Emergence of Emergence. Boston, MA: MIT Press, (pp. 111-150).

10. Deacon, T. W. (2006). Reciprocal linkage between self-organizing processes is sufficient for self-reproduction and evolvability. Biological Theory, 1(2), 136-149.

11. Deacon, T. W. (2012). Incomplete Nature: How Mind Emerged from Matter. New York, NY: W.W. Norton and Company.

12. Deacon, T. W., \& Koutroufinis, S. A. (forthcoming). Information, complexity and dynamic depth. Information.

13. Eigen, M., \& Schuster, P. (1979). The Hypercycle - A Principle of Natural SelfOrganization. Heidelberg: Springer.

14. Fellermann, H., Rasmussen, S., Ziock, H.-J., \& Solé, R. V. (2007). Life cycle of a minimal protocell—a dissipative particle dynamics study. Artificial Life, 13(4), 319-345.

15. Getling, A. V. (1998). Rayleigh-Bénard convection: structures and dynamics, vol. 11. World Scientific.

16. Haken (2006). Information and self-organization: a macroscopic approach to complex systems. Springer.

17. Harder, M., \& Polani, D. (2013). Self-organizing particle systems. Advances in Complex Systems, 16. 
18. Jost, L. (2006). Entropy and diversity. Oikos, 113, 363-375.

19. Joyce, G. F. (1989). RNA evolution and the origins of life. Nature, 338, 217-224.

20. Kauffman, S. A. (1993). Origins of Order: self-organization and selection in evolution. New York, NY: Oxford University Press.

21. Kauffman, S. A. (2011). Approaches to the origin of life on earth. Life, 1(1), 34-48.

22. Kullback, S., \& Leibler, R. (1951). On information and sufficiency. The Annals of Mathematical Statistics, 22(1), 79-86.

23. Langton, C. G. (1986). Studying artificial life with cellular automata. Physica D, 22, $120-149$.

24. Maturana, H. R., \& Varela, F. J. (1980). Autopoiesis and cognition. Boston, MA: Reidel.

25. McMullin, B., \& Varela, F. J. (1997). Rediscovering computational autopoiesis. In P. Husbands, \& I. Harvey, eds., Fourth European Conference on Artificial Life. Cambridge, MA: MIT Press, (pp. 38-48).

26. Plasson, R., Brandenburg, A., Jullien, L., \& Bersini, H. (2011). Autocatalysis: At the root of self-replication. Artificial Life, 17(3), 219-236.

27. Polani, D. (2008). Foundations and formalizations of self-organization. Advances in Applied Self-Organizing Systems, 19-37.

28. Prigogine, I., \& Stengers, I. (1984). Order Out of Chaos. New York, NY: Bantam Books.

29. Rosen, R. (1991). Life itself: A Comprehensive Inquiry into the Nature, Origin, and Fabrication of Life. New York: Columbia University Press. 
30. Shannon, C. E. (1948). A mathematical theory of communication. Bell Systems Technical Journal, 27, 379-423.

31. Thompson, E. (2010). Mind in Life. Cambridge, MA: Harvard University Press.

32. Tyson, J. L. (1976). The Belousov-Zhabotinski Reaction. Berlin: Springer-Verlag.

33. Varela, F. J., Maturana, H. R., \& Uribe, R. (1974). Autopoiesis: The organization of living systems, its characterization and a model. BioSystems, 5, 187-196.

34. von Neumann, J. (1966). The Theory of Self-Reproducing Automata. Chicago, IL: University of Illinois Press. 


\section{Appendix: Model Description}

A non-toroidal two-dimensional $10 \times 10$ tile grid is used as a discrete model of a reactiondiffusion system containing seven different types of particles ( $A$ to $G$ ). At the start of a simulation run, the particles are distributed randomly over the grid. Then, for each time step, each particle may

1. move to a neighboring tile;

2. collide and react with other particles in the same tile;

3. break up.

These steps are described in more detail below.

\section{Movement}

Random movement caused by bouncing agains the edges of the grid, or particle-to-particle collision, is approximated by randomly directed movement to a horizontally or vertically neighboring tile with $P_{\text {movement }}=0.1$ for each particle or crystal.

\section{Collision}

Self-assembly of $G^{n}$ crystals:

$$
G^{n}+G \rightarrow G^{n+1}
$$

with reaction probability $P_{g}^{+}=\gamma^{+} \in[0,1]$ with $n \geq 1$. Reciprocal catalysis of particle types $A$ to $F$

$$
A+B \stackrel{F}{\rightarrow} C
$$


and

$$
D+E \stackrel{C}{\rightarrow} F,
$$

with $P_{r}^{+}=\left(1+\exp \left[\varrho^{+}\right]\right)^{-(1+n)^{-2}}, \varrho^{+} \in \mathbb{R}$ and $n$ the number of catalysts present at tile $i$ (i.e. $n=\left|F_{i}\right|$ for the former reaction, and $n=\left|C_{i}\right|$ for the latter). For the experiments in sections 6 and 7, self-assembly and reciprocal catalysis are combined:

$$
D+E \stackrel{C}{\rightarrow} F+G
$$

Containment is modeled by modifying the attachment reaction for self-assembly:

$$
G^{n}(k C, m F)+G+p C+q F \rightarrow G^{n+1}((k+p) C,(m+q) F) .
$$

The experiments in section 7 feature a containment capacity $c$, representing the maximum number of catalysts a crystal may contain:

$$
G_{c}^{n}(k C, m F)+G+p C+q F \rightarrow G_{c}^{n+1}\left(k^{\prime} C, m^{\prime} F\right)+p^{\prime} C+q^{\prime} F
$$

with $k^{\prime}+m^{\prime} \leq c, k^{\prime}+p^{\prime}=k+p$ and $m^{\prime}+q^{\prime}=m+q$. If $k+m+p+q>c$, the order in which catalyst are contained is determined at random.

\section{Breakup}

Particles types $G, G^{n}, C$ and $F$ have a probability of breaking up:

$$
G^{n} \rightarrow G^{n-1}+G
$$

with $\gamma^{-} \in \mathbb{R}$ and $P_{g}^{-}=\left(1+\exp \left[\gamma^{-}\right]\right)^{-n}$. If the crystal contains catalysts, they are released upon detachment (irrespective of the containment capacity $c$ ): 


$$
G^{n}(k C, m F) \rightarrow G^{n-1}+G+k C+m F
$$

Furthermore,

$$
C \rightarrow A+B
$$

and

$$
F \rightarrow D+E
$$

with reaction probability $P_{r}^{-}=\varrho^{-} \in[0,1]$. In sections 6 and $7, G$ particles are removed from the grid with $P_{g}^{-}=\left(1+\exp \left[\gamma^{-}\right]\right)^{-1}$. 